\title{
Étude épidémiologique sur les extractions dentaires chez les patients sous AVK en Ille-et-Vilaine
}

\section{Murielle Massot, Fabrice Clipet, Nora Alno, Julien Garnier, Bertrand Köhler, Gilbert De Mello*}

Service de Chirurgie buccale, CHU Rennes, Faculté d'Odontologie, Université de Rennes 1, Université Européenne de Bretagne, France

\section{(Med Buccale Chir Buccale 2009;15:5-15)}

Mots clés :

chirurgie buccale / antivitamines $\mathrm{K} /$ étude épidémiologique / extraction dentaire / hémorragie
Key words: oral surgery / oral anticoagulant treatment / epidemiological study / dental extraction / hemorrhage

\begin{abstract}
Résumé - La Société Française de Médecine buccale et de Chirurgie buccale (SFMBCB) et la Société Française de Cardiologie (SFC) ont rédigé en 2006 des recommandations relatives à la prise en charge des patients sous anticoagulants oraux. Désormais, lors de la prise en charge dans une pratique de ville, le traitement AVK ne doit plus être modifié avant des extractions dentaires, si l'INR est stable et inférieur ou égal à 3. Bien entendu dans ce cas, une hémostase locale de qualité, se composant de la mise en place d'un comblement intra-alvéolaire avec un biomatériau hémostatique résorbable, de sutures en points séparés et de la prescription de compressions intermittentes, doit être réalisée. Un contrôle postopératoire à 72 heures est préconisé.

L'étude épidémiologique menée auprès des chirurgiens dentistes d'Ille-et-Vilaine en 2007 a pour objectif d'évaluer l'application de ces pratiques.

Le bilan pré-opératoire, la technique d'hémostase mise en œuvre et les contrôles postopératoires ont été comparés aux recommandations de la SFMBCB et de la SFC.
\end{abstract}

\begin{abstract}
Epidemiological study of management of patients with oral anticoagulant treatment in Ille-et-Vilaine, France. Until recently, there was no protocol established relating to the management of patients with oral anticoagulant treatment before an act of oral surgery. Following the last studies completed on this subject, the French Society of Oral Medicine and Oral Surgery (SFMBCB) and the French Society of Cardiology (SFC) decided to write the new recommendations in 2006 relating to the management of these hypocoagulated patients. From now onwards, the oral anticoagulation treatment will not have to be changed before dental extractions, if INR is stable and less than 3. A local haemostasis will have to be accomplished with quality: filling of the socket, sutures in separated points, intermittent compressions and postsurgical control on the third day.

The objective of the study of epidemiology is to give an account, of 2007, of the practices of the dental surgeons for the patients with oral anticoagulant treatment in Ille-et-Vilaine, France. And thus, it will assess different used protocols, techniques of haemostasis, with possible complications, and the sources of information of the practitioners of Ille-et-Vilaine. These data will be compared with recommendations of SFMBCB and SFC.
\end{abstract}

En odontologie, la prise en charge des patients sous traitement anticoagulant, et en particulier sous antivitamines $\mathrm{K}$ (AVK) est en évolution. Actuellement, environ $1,5 \%$ de la population française est traité par AVK, soit environ 600000 personnes. La majorité de ces patients est représentée par

\footnotetext{
^Correspondance :

Gilbert De Mello, gilbert.de-mello@univ-rennes1.fr
}

des personnes âgées, très souvent polymédiquées, donc fragiles [1-3].

Les AVK sont utilisés pour prévenir le risque thromboembolique. Leur complication majeure en odontologie est représentée par le risque hémorragique. Lors des actes de chirurgie buccale, le praticien doit prendre en compte ces prescriptions afin d'éviter une hémorragie per-opératoire ou postopératoire, tout en maintenant un état d'hypocoagulabilité compatible avec l'affection dont souffre le patient. Le risque 
hémorragique lié à l'acte quotidien et au traitement par AVK doit être évalué avant chaque intervention de chirurgie buccodentaire invasive.

Depuis 2006, la Société Française de Cardiologie (SFC) et la Société Francophone de Médecine buccale et de Chirurgie buccale (SFMBCB) recommandent la non modification du traitement anticoagulant avant un acte de chirurgie buccale [4]. En effet, le traitement d'hypocoagulation a pour but de diminuer le risque thromboembolique; l'arrêter peut avoir des conséquences létales pour le patient $[2,5]$. La réalisation d'un relais avec l'héparine perturbe le traitement hypocoagulant qui est par la suite difficile à ajuster et à stabiliser. La mise en place d'un relais augmente le risque thromboembolique [6] et n'empêche pas les hémorragies postopératoires [7].

Le maintien du traitement par AVK est donc l'option thérapeutique de choix dans le respect d'un protocole opératoire strict : INR stable et inférieur à 4 pour une prise en charge hospitalière ou inférieur ou égal à 3 pour une prise en charge en pratique de ville, et réalisation d'une hémostase locale systématique, utilisation de colle biologique et/ou d'agent antifibrinolytique en cas d'interventions chirurgicales hémorragiques. Il n'existe pas dans la littérature actuelle de complications graves avec le maintien du traitement anticoagulant lors d'une intervention de chirurgie buccale.

Afin d'évaluer les pratiques actuelles de prise en charge des patients sous AVK, une étude épidémiologique des pratiques des chirurgiens dentistes d'Ille-et-Vilaine a été effectuée à partir d'un questionnaire adressé à tous les praticiens. Le recueil des données a été réalisé en 2007.

\section{Matériel et Méthodes}

\section{Méthode}

Durant le mois de mai 2007, un questionnaire a été envoyé par courrier aux 730 chirurgiens dentistes d'Ille-et-Vilaine inscrits sur l'annuaire des chirurgiens dentistes du Conseil départemental de l'Ordre. Tous les chirurgiens dentistes d'Illeet-Vilaine ayant une activité omnipratique ou ayant une activité de chirurgie exclusive ont été inclus dans l'étude. Les réponses ont ensuite été traitées avec le logiciel informatique NeoOffice.

\section{Matériel}

Pour réaliser cette étude, nous avons utilisé : un courrier, un questionnaire et un logiciel informatique. Le courrier a été adressé à chaque praticien. A l'intérieur se trouvait un courrier informatif décrivant l'étude, un questionnaire et une enveloppe timbrée avec l'adresse pré-inscrite. Le questionnaire comprenait 16 questions qui reprenaient les différents protocoles opératoires mis en place lors de la réalisation d'un acte chirurgical au cabinet dentaire. La collecte des résultats s'est déroulée entre mai 2007 et août 2007.

\section{Resultats}

Sur 730 questionnaires envoyés, 312 ont été renvoyés soit $42,7 \%$ de réponses. Parmi ces résultats, 12 sont inexploitables: 5 praticiens ont retourné le courrier en précisant qu'ils exerçaient en tant qu'orthodontistes, 4 en précisant qu'ils n'exerçaient plus, enfin 3 questionnaires n'ont pas été remplis. Sur les 300 questionnaires exploitables, l'échantillon est composé de 155 femmes $(51,7 \%)$ et de 145 hommes $(48,3 \%)$. La date d'installation de ces confrères est comprise entre 1966 et $2007 ; 52$ praticiens ne sont pas encore installés : ils travaillent en qualité de collaborateur.

Les praticiens ne prenant pas en charge les patients sous AVK représentent 43,3\% de l'échantillon (130/300) : 89 hommes $(61,3 \%)$ et 41 femmes $(26,4 \%)$. Leurs patients sont alors adressés au médecin traitant $(3,8 \%)$, au CHU ou à l'hôpital $(23,1 \%)$ ou encore à un cabinet dentaire spécialisé libéral $(40,8 \%) .32,3 \%$ des praticiens ne prenant pas en charge les patients sous AVK ne se prononcent pas sur ce sujet.

170 praticiens $(56,7 \%)$ prennent en charge les patients sous AVK lors d'actes sanglants : 56 praticiens hommes $(38,6 \%)$ et 114 praticiens femmes $(73,6 \%)$. Sur ces 170 praticiens, $27(15,9 \%)$ choisissent de ne pas arrêter le traitement anticoagulant dans la plupart des cas, sinon ils demandent un relais ou un arrêt du traitement AVK (ces praticiens appartiennent à la catégorie « autres » dans les tableaux et figures). $28(16,5 \%)$ choisissent régulièrement de ne pas arrêter le traitement AVK.

Parmi ces 28 praticiens (22 praticiens femmes et 6 praticiens hommes) respectant le protocole de la SFMBCB, 3 les adressent en cas de haut risque hémorragique : 2 vers un cabinet dentaire libéral ayant une activité exclusive de chirurgie buccale et 1 au médecin traitant.

Sur ces 28 praticiens, 17 ont indiqué la valeur limite de l'INR pour laquelle ils réalisent un acte sanglant : les valeurs se situent entre 2,5 et 4,5 avec un INR moyen de 3. La Fig. 1 montre la répartition des praticiens prenant en charge les patients sous AVK selon leur sexe et leur date d'installation, et indique également comment ces praticiens assurent la prise en charge. Sur ces 28 praticiens, 21 demandent un INR : 4 demandent un INR seul et 11 demandent un ou plusieurs examens supplémentaires. Sur les 21 praticiens demandant un INR sans autre examen complémentaire, 19 réalisent une hémostase locale de niveau 1 comprenant la mise en place d'un matériau de comblement intra-alvéolaire, la réalisation de suture et la pratique d'une compression. Parmi les 19 praticiens réalisant une hémostase locale de niveau 1, 10 réalisent des sutures avec du fil résorbable et 9 avec du fil non résorbable. De plus, ils sont 13 à utiliser des compresses humides, imbibées d'acide tranexamique, et 6 à utiliser des compresses sèches. Sur ces 19 praticiens, 17 réalisent un ou plusieurs contrôles postopératoires : 15 minutes après l'acte pour 13 praticiens, 48 heures après pour 3 praticiens et 10 praticiens une semaine après. Au final, 19 praticiens sur 300 suivent les recommandations rédigées par la SFMBCB, soit $11,2 \%$ des praticiens prenant en charge les patients sous AVK. 


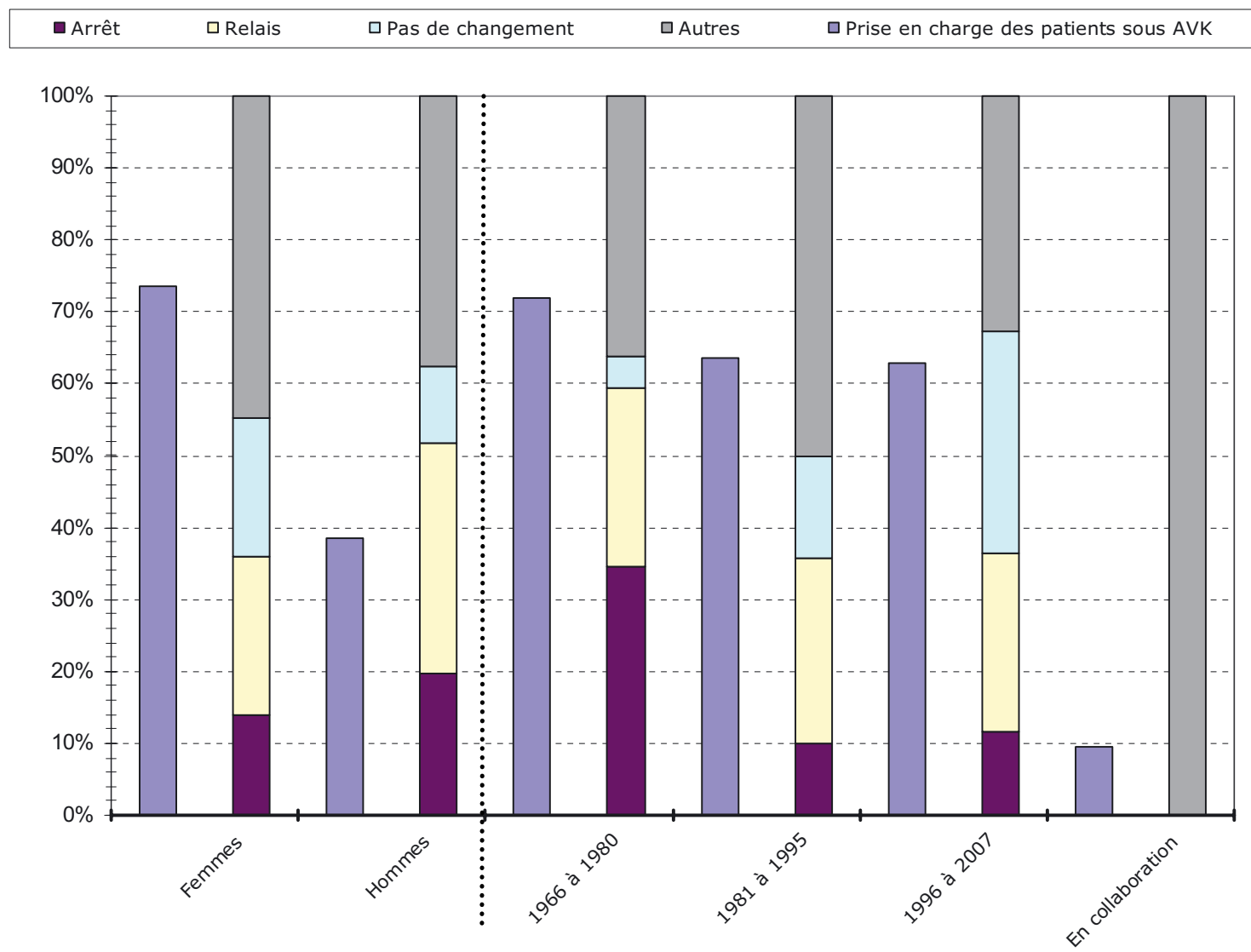

Fig. 1. Protocole de prise en charge des patients sous AVK, selon le sexe et la date d'installation du praticien.

Fig. 1. Management of patients with oral anticoagulant treatment according to the sex and the date of installation.

Parmi les 170 praticiens traitant des patients sous AVK, 27 $(15,9 \%)$ demandent l'arrêt du traitement AVK, 43 (25,3\%) demandent le relais du traitement AVK et 72 (42,9\%) utilisent divers protocoles. En fonction du cas, cinq de ces 72 praticiens adoptent l'arrêt, le relais ou la poursuite sans modification du traitement AVK; les 67 autres praticiens optent pour l'une des solutions suivantes : arrêt ou relais (44 praticiens), arrêt ou pas de changement ( 6 praticiens) ou encore relais ou poursuite sans modification du traitement AVK (17 praticiens).

$29,4 \%(50 / 170)$ des praticiens prenant en charge des patients sous AVK ont déjà rencontré une ou plusieurs complications liées au traitement par les AVK. Ces complications peuvent être peropératoires $(5,9 \%)$ ou postopératoires $(27,1 \%)$. Il peut s'agir d'hémorragies postopératoires $(40 \%)$, de caillots extra-alvéolaires (36,5\%) ou bien encore d'hématomes $(23,5 \%)$ (Tab. III).

$30,3 \%(91 / 300)$ des praticiens ayant renvoyé le questionnaire s'estiment suffisamment informés sur les dernières recommandations en vigueur concernant les protocoles de prise en charge des patients sous AVK, leurs sources d'information sont diverses. Par contre, $63,7 \%$ des praticiens interrogés considèrent avoir besoin d'une formation supplémentaire pour la prise en charge des patients sous AVK au cabinet dentaire (Fig. 2)

\section{Discussion}

La prévalence des patients traités par AVK est en augmentation constante. Ce sont des patients à risque thromboembolique que le chirurgien dentiste est amené à rencontrer dans son exercice quotidien [8]. Les praticiens appréhendent souvent la prise en charge de ces patients en raison du risque hémorragique et il n'existait pas de consensus clairement établi. Chacun adoptait son propre protocole entre l'arrêt complet du traitement anticoagulant et à la poursuite sans modification du traitement. A la suite des dernières études menées sur ce sujet $[3,5,9-19]$, la SFMBCB et la SFC ont rédigé de nouvelles recommandations en 2006, puis se sont efforcés de les diffuser afin que l'information parvienne aux différents intervenants - cardiologue, médecin traitant et odontologiste - pour améliorer la coordination et la prise en charge des patients afin de réduire au minimum le risque hémorragique et le risque thromboembolique. Il ya encore des discordances au sein des professionnels puisque au moins un praticien a signalé qu'il était en désaccord avec le cardiologue qui voulait encore systématiquement stopper le traitement anticoagulant pour réaliser un relais héparinique avant des extractions dentaires. 
$\square$ Internet $\square$ Revues $\square$ Formation continue $\quad$ Congrès $\square$ Autres $\square$ Besoin formation $\square$ Connaissance des recommandations

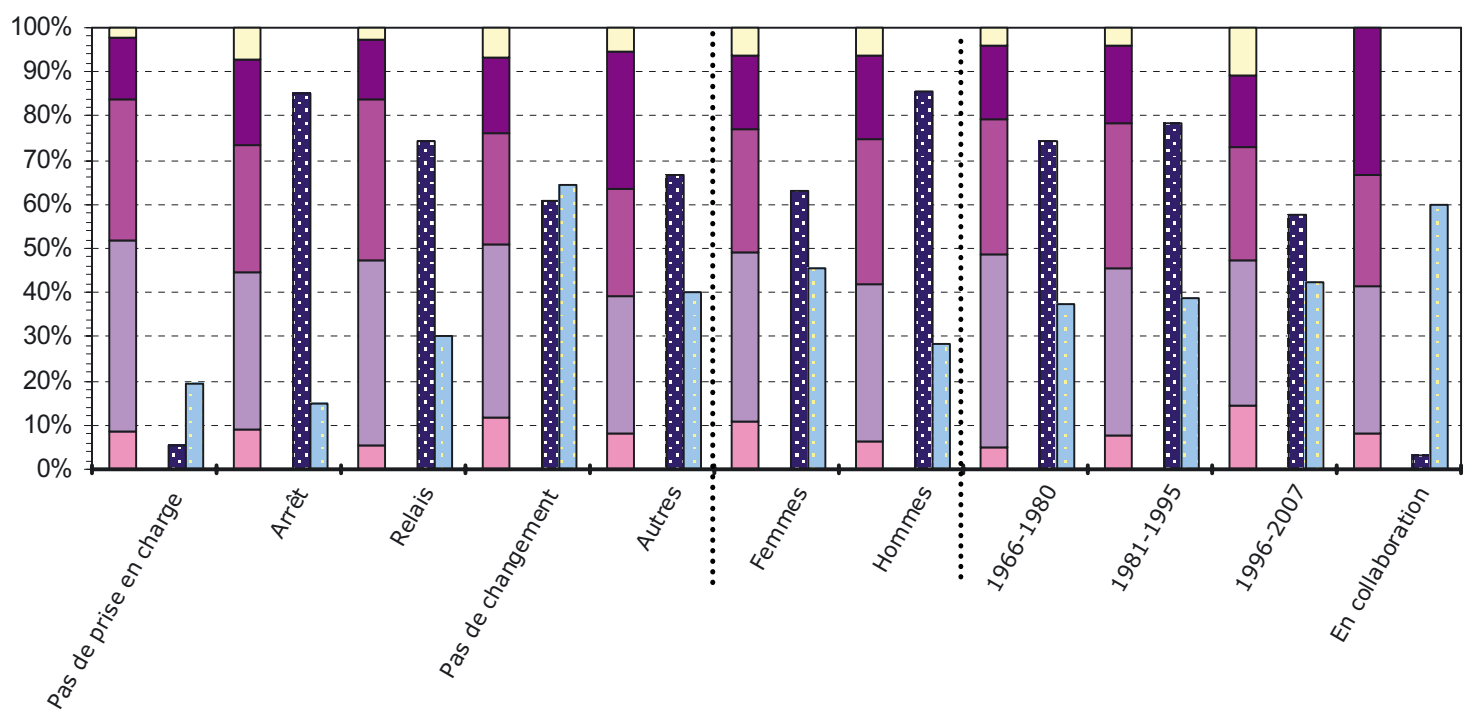

Fig. 2. Connaissance des recommandations sur la prise en charge des patients sous AVK et mode de transmission selon le sexe et la date d'installation du praticien.

Fig. 2. Knowledge of the recommendations according to the sex and the date of installation.

Tableau I. Coût d'une extraction dentaire chez le patient sous AVK [25].

Table I. Price of one extraction in patient with oral anticoagulant treatment according to the INR.

\begin{tabular}{|l|c|c|}
\hline Technique de prise en charge & Patient A & Patient B \\
\hline Pas de modification du traitement AVK & $152 €$ & $223 €$ \\
\hline Relais aux HNF sans hospitalisation selon le protocole mis en place au CHU de Rennes & $328 €$ & $434 €$ \\
\hline Relais aux HBPM sans hospitalisation selon le protocole mis en place au CHU de Rennes & $310 €$ & $425 €$ \\
\hline Arrêt du traitement AVK & $221 €$ & $230 €$ \\
\hline
\end{tabular}

Coût selon l'attitude de prise en charge :

Patient $A$ : risque hémorragique modéré (INR stable aux environs de 2)

Patient $B$ : risque hémorragique important (INR stable aux environs de 3,5)

Chez les patients sous AVK, la fréquence des accidents hémorragiques graves, d'origine bucco-dentaire ou non, varie de 1,3 à $10 \%$, soit entre 1,2 et $5,6 \%$ par an et par malade. Dans toute la littérature étudiée, il n'y a pas de complications graves ni de décès survenant après des extractions dentaires lorsque le traitement AVK était maintenu. Il est donc désormais démontré qu'il ne faut pas modifier systématiquement le traitement AVK avant une intervention de chirurgie buccale $[17,19,20]$.

Les recommandations de la SFMBCB préconisent de ne pas arrêter le traitement anticoagulant pour réaliser des extractions dentaires si celui-ci est équilibré et bien contrôlé avec un INR inférieur à 3 au moment de l'intervention. De plus, cette nouvelle approche diminue le surcoût de prise en charge thérapeutique (Tab. I). Il est important que les praticiens aient connaissance de ce coût puisque les dépenses de notre système de santé sont maintenant une préoccupation majeure. En effet, le relais héparinique ou l'arrêt du traitement AVK entraînent un surcoût non négligeable. Ainsi l'utilisation
d'HBPM nécessite une infirmière à domicile, l'utilisation d'héparine nécessite une hospitalisation. L'arrêt du traitement AVK impose de réaliser des examens biologiques pour retrouver un INR stable.

Lorsque l'INR est stable et inférieur à 3, le praticien doit réaliser une hémostase de niveau $1:$ matériau de comblement intra-alvéolaire, sutures et compressions intermittentes. Ces compressions peuvent être réalisées avec des compresses imbibées d'acide tranexamique (Exacyl $\left.{ }^{\circledR}\right)$. C'est un antifibrinolytique qui favorise l'hémostase pour un faible coût. Boréa et al. ont montré, dans une étude randomisée en double aveugle, qu'il n'y avait pas de différence significative dans le saignement après extraction entre un patient dont le traitement AVK n'est pas modifié et recevant par voie locale de l'acide tranexamique et un patient dont le traitement AVK a été diminué [21].

Notre étude a essayé de dresser un premier état des lieux de la prise en charge de ces patients en Ille-et-Vilaine et de préciser les différents types d'approche. La presque 
Tableau II. Coût des examens complémentaires pour 1000 extractions. Table II. Price of biological analysis for 1000 extractions.

\begin{tabular}{|c|c|c|c|c|}
\hline Examen & Prix unitaire & $\%$ prescription & Coût pour 1000 extractions & Recommandations SFMBCB \\
\hline NFS & $14,31 €$ & $37,4 \%$ & $5351,94 €$ & $0 €$ \\
\hline NP & $10,26 €$ & $8,8 \%$ & $902,88 €$ & $0 €$ \\
\hline TCA & $11,61 €$ & $19,8 \%$ & $2298,78 €$ & $0 €$ \\
\hline TP/INR & $10,26 €$ & $71,4 \%$ & $7325,64 €$ & $10260 €$ \\
\hline TOTAL & & & $15879,24 €$ & $10260 €$ \\
\hline
\end{tabular}

totalité des chirurgiens dentistes de ce département a reçu le questionnaire et le grand nombre de réponses $(42,7 \%$ de réponses) donne un bon échantillon de la population de praticiens exerçant dans ce département.

En Ille-et-Vilaine, 56,7\% des praticiens prennent en charge les patients sous AVK. La proportion augmente de 10 points $(66,5 \%)$ si l'on retire les praticiens collaborateurs. Il est surprenant de constater que les praticiens non installés, c'està-dire sortant du cycle universitaire et susceptibles de maîtriser les dernières recommandations en vigueur, ne prennent que très peu en charge ces patients. En effet, seuls 9,6 \% d'entre eux réalisent cette prise en charge. On peut s'interroger sur ce chiffre et se demander pourquoi les praticiens nouvellement formés hésitent à prendre en charge ces patients au début de leur exercice professionnel?

Le pourcentage de praticiens femmes prenant en charge ces patients est significativement supérieur à celui des praticiens hommes : $73,5 \%$ contre $38,6 \%$. Les praticiens ne prenant pas en charge ces patients les envoient plus facilement dans un cabinet libéral ayant une activité de chirurgie buccale exclusive plutôt qu'à une structure hospitalière. Le médecin n'est que peu consulté sur cette question $(3,8 \%)$. Le nombre de praticiens ne prenant pas en charge les patients sous AVK devrait être comparé à celui des praticiens ne réalisant pas d'actes chirurgicaux dans leur pratique courante mais il n'y a pas de statistique disponible.

L'attitude thérapeutique recommandée en première intention au cabinet dentaire, et rappelée par la SFMBCB et la SFC en 2006, est la réalisation des extractions dentaires en poursuivant le traitement AVK à condition que celui-ci soit stable et contrôlé avec un INR inférieur à 3. Selon les données de notre étude, la majorité de praticiens continue à pratiquer un relais du traitement AVK par l'héparine (HNF ou HBPM) préférant diminuer le risque hémorragique per-opératoire en augmentant le risque thromboembolique, souvent mal connu et sous-estimé. Rappelons que la littérature répertorie de nombreux cas documentés d'accidents hémorragiques ou thromboemboliques graves après arrêt ou relais du traitement AVK et qu'à l'inverse il n'existe pas de cas d'accident hémorragique grave décrit lorsque le traitement AVK est maintenu $[5,14,16-$ $19,22]$. Cependant, il y a encore peu d'études comparatives sur le risque de complications en fonction du protocole utilisé. On remarque qu'une très grande partie des praticiens utilise différents protocoles en fonction du risque hémorragique ren- contré, mais cette étude ne permet pas de dire dans quel cas ils utilisent l'un ou l'autre des protocoles.

Les praticiens maintenant systématiquement le traitement AVK représentent $16,5 \%$ des chirurgiens dentistes prenant en charge ces patients. Parmi ces derniers, les praticiens femmes semblent adopter ce choix thérapeutique plus souvent que les praticiens hommes $(19,3 \%$ contre $10,7 \%)$, mais la différence n'est pas significative.

Les chirurgiens dentistes ont été divisés en trois groupes selon leur date d'installation : de 1966 à 1980, de 1981 à 1995 et de 1996 à 2007. La prise en charge évolue parallèlement à la date d'installation. Le groupe le plus ancien (1966 à 1980) réalise plus d'arrêts du traitement AVK que les autres groupes. La pratique du relais est uniformément répartie dans les trois groupes d'âge. L'arrêt est quasiment abandonné par les jeunes praticiens par rapport aux praticiens anciennement installés. Cependant, dans les trois groupes d'âge, on note que le relais est encore trop souvent réalisé. Une campagne d'information devrait être menée pour informer les praticiens des dernières recommandations, autant au niveau des odontologistes que des cardiologues et des médecins traitants.

Une consultation et un bilan pré-opératoire sont recommandés avant de réaliser un acte sanglant. Celui-ci passe d'abord par la connaissance des antécédents médicaux du patient et la mise à jour de son dossier. Le praticien doit s'informer sur le traitement médical du patient et les derniers examens complémentaires, en particulier la dernière valeur de L'INR. Une bonne coordination entre les divers praticiens est nécessaire et ils doivent décider ensemble d'une stratégie commune; la responsabilité appartient à l'ensemble de ces acteurs : cardiologue, médecin traitant, chirurgien dentiste.

Pour obtenir le consentement éclairé du patient on doit clairement l'informer du risque surajouté lié au traitement AVK. Il est clairement établi que le niveau d'hypocoagulation doit être évalué par un INR dans les 24 heures précédant l'intervention chirurgicale. Il est inutIlle de réaliser une NFS ou un TCA lorsque le traitement est maintenu. Il apparaît ainsi que les examens biologiques ne sont pas prescrits en Ille-et-Vilaine. Pour illustrer le coût de ces examens, prenons comme exemple le coût de mille extractions en Ille-et-Vilaine données par les praticiens, chaque extraction étant réalisée chez des patients différents (Tab. II). En suivant les recommandations de la SFMBCB et la SFC, l'économie ainsi réalisée pour mille extractions serait de $5619,24 €$ si l'ensemble des praticiens s'y conformait. Le rapport coût/sécurité est donc 
Tableau III. Complications rencontrées en fonction du protocole utilisé.

Table III. Post-operative complications according to the protocol of management.

\begin{tabular}{|c|c|c|c|c|}
\hline & Arrêt & Relais & Pas de changement & Autres \\
\hline Total praticiens & 27 & 43 & 28 & 72 \\
\hline Complications & $18,2 \%$ & $32,6 \%$ & $42,9 \%$ & $26,4 \%$ \\
\hline Per opératoires & $3,1 \%$ & $4,6 \%$ & $7,1 \%$ & $6,9 \%$ \\
\hline Postopératoires & $18,2 \%$ & $30,2 \%$ & $32,1 \%$ & $23,6 \%$ \\
\hline Hémorragie & $11,1 \%$ & $25,6 \%$ & $21,4 \%$ & $18,1 \%$ \\
\hline Caillot extra- alvéolaire & $14,1 \%$ & $18,0 \%$ & $25 \%$ & $16,7 \%$ \\
\hline Hématome & $7,1 \%$ & $11,3 \%$ & $25 \%$ & $8,3 \%$ \\
\hline
\end{tabular}

positif si les recommandations de la SFMBCB et de la SFC sont suivies.

Selon les recommandations de la SFMBCB, les soins dentaires peuvent être pratiqués avec un INR se situant entre 2 et 3 pour les patients sous AVK en prévention des embolies systémiques et entre 3 et 3,5 pour les patients sous AVK pour les patients ayant une prothèse valvulaire mécanique ou une valvulopathie mitrale sévère avec facteurs favorisants. En Ille-et-Vilaine, pour les patients pris en charge, l'INR moyen est de 2,81; l'INR moyen des patients pris en charge par les praticiens qui ne pratiquent pas de changement du traitement AVK est de 2,94, ce qui respecte la valeur préconisée de la SFMBCB dans les recommandations. Les praticiens qui ne soignent pas les patients avec un INR supérieur à 2 demandent l'arrêt du traitement AVK. L'argumentation des recommandations de la SFMBCB rappelle que de nombreuses études [14, 23, 24] démontrent que les avulsions dentaires peuvent être réalisées sans modifier le traitement AVK avec un INR inférieur à 4 si on réalise une hémostase locale. Pour prévenir les risques hémorragiques lors des actes chirurgicaux chez les patients sous AVK, on doit systématiquement réaliser une hémostase locale. La maîtrise de celle-ci est fondamentale, l'obtention d'une hémostase locale efficace et son contrôle constituent une étape essentielle de l'acte chirurgical. Le praticien dispose d'un arsenal de moyens et de matériaux d'hémostase locale. 95,3\% des praticiens interrogés disent utiliser un moyen d'hémostase locale. Cependant l'analyse des résultats montre que seuls $72,2 \%$ réalisent l'hémostase conseillée par les recommandations de la SFMBCB : comblements intra-alvéolaires, sutures et compressions intermittentes avec de l'Exacyl ${ }^{\circledR}$. On constate que les comblements les plus utilisés sont les éponges de gélatine et le collagène dénaturé : elles représentent $66,7 \%$ des prescriptions $\left(21,7 \%\right.$ des praticiens utilisent du Curaspon ${ }^{\circledR}$, $13,8 \%$ du Pangen ${ }^{\circledR}$ et $31,1 \%$ d'autres produits comme l'Etik Collagène ${ }^{\circledR}$ ou l'Hemocollagène ${ }^{\circledR}$ ). Le Surgice ${ }^{\circledR}$ reste également très utilisé : $43,5 \%$. Or, si les recommandations de la SFMBCB précisent qu'un agent hémostatique local résorbable doit être mis en place dans les alvéoles, elles spécifient également que le contact osseux direct avec l'oxycellulose est contre-indiqué [4]. En ce qui concerne les sutures, $81,8 \%$ des praticiens en réalisent. Le protocole des recommandations de la SFMBCB précisent que les plaies doivent être suturées avec des points de suture séparés, mais ne conseille pas spécifiquement l'utilisation de fil résorbable ou non résorbable. 92,3\% des praticiens font réaliser des compressions à leurs patients. La plupart des praticiens utilise des compresses sèches $(70 \%)$; pour la majorité des praticiens $(67,1 \%)$, la compression doit durer au moins 10 minutes, conseil retrouvé dans les recommandations de la SFMBCB. Cependant cellesci optent pour la réalisation postopératoire de compressions à l'aide d'une compresse imbibée d'acide tranexamique. Un suivi du patient est nécessaire pour prévenir le risque hémorragique et pouvoir intervenir rapidement en cas de complications. Ainsi $51,8 \%$ des praticiens réalisent un contrôle 15 minutes après l'acte, 17,6 $\% 48$ heures après et $40 \%$ une semaine après.

Les recommandations de la SFMBCB précise que les patients sous traitement AVK doivent être pris en charge comme les patients en chirurgie ambulatoire : "Ils doivent être accompagnés, habiter à moins d'une heure d'une structure de soins adaptée à l'acte, être aptes à observer les prescriptions médicales et avoir une compréhension suffisante de ce qui leur est proposé. Dans le cas où ces critères ne sont pas remplis, le geste se fera au cours d'une hospitalisation ». Les praticiens doivent assurer la meilleure prise en charge possible et ils ont l'obligation d'assurer une continuité de soins dans des conditions de sécurité optimales [25].

Les traitements AVK comportent toujours de nombreuses complications dont les plus fréquentes et les plus graves sont les accidents hémorragiques. En Ille-et-Vilaine, 29,4\% des chirurgiens dentistes estiment avoir déjà rencontré des complications. Mais le nombre de complications postopératoires ne prend-il pas également en compte les patients qui ne sont pas sous AVK?

Le relais par l'héparine ou par les HBPM comporte des inconvénients indéniables. $24,7 \%$ des praticiens prenant en charge des patients sous AVK optent pour ce choix thérapeutique. Lors du relais, le passage d'une substance à une autre implique un monitorage délicat de l'hypocoagulation. De plus, le retour de l'activité des AVK au seuil thérapeutique se fait en plusieurs jours rendant le risque thromboembolique non négligeable [6]. Il existe une tendance à sous-estimer le risque de complications thrombotiques et à le minimiser en comparaison du risque hémorragique. Ceci est dû au fait que ces complications surviennent à distance de l'acte et ne sont pas 
toujours connues du chirurgien dentiste ou du médecin traitant. A ce problème se surajoute le risque de thrombocytopénie induite par l'héparine. Parmi les praticiens utilisant ce protocole, $32,6 \%$ ont déjà observé des complications hémorragiques. Le relais héparinique doit être réservé aux patients à haut risque thromboembolique et à INR élevé, en particulier les porteurs de prothèse valvulaire mécanique et chez les patients non coopérants [4].

Pour les patients sous AVK, 15,9\% des praticiens réalisent l'arrêt complet du traitement AVK. Cette stratégie paraît encore largement répandue et semble offrir la meilleure protection contre la survenue d'une hémorragie postopératoire. Cependant, la diminution pré-opératoire de l'INR est souvent imprévisible et le retour à un INR stable postopératoire compliqué. Devani et al. [5] ont montré dans une étude sur 133 extractions dentaires qu'il s'est révélé impossible pour $50 \%$ des patients de baisser l'INR entre 1,5 et 2,1 dans les 48 heures précédant les extractions dentaires. De plus, Wahl [17] a montré dans une étude rétrospective auprès de 950 patients hypocoagulés et soumis à 2400 actes de chirurgie buccale que le risque thrombo embolique était trois fois plus élevé chez les patients pour lesquels l'hypocoagulation était stoppée que le risque hémorragique chez ceux pour lesquels l'hypocoagulation n'avait pas été arrêtée. Notons que Wahl rapporte quatre décès parmi les patients chez lesquels le traitement AVK avait été arrêté [17]. Une autre série comportant 542 extractions dentaires, effectuées avec arrêt du traitement AVK et sans relais héparinique, rapporte $1 \%$ de complications thromboemboliques [26]. En Ille-et-Vilaine, $18,5 \%$ des praticiens arrêtant le traitement AVK estiment avoir déjà rencontré des complications. Un praticien relate le fait que l'un de ses patients a fait un accident vasculaire cérébral après qu'on lui ait stoppé son traitement anticoagulant pour des extractions qu'il a réalisées sans complication; le patient est décédé un an plus tard. On s'étonne que certains praticiens de cette catégorie décrivent des caillots, des hémorragies ou des hématomes comme complications alors que théoriquement le risque hémorragique devrait être nul si on arrête le traitement AVK.

Dans la littérature, il n'existe pas de cas documenté relatant une hémorragie grave après une extraction dentaire chez un patient dont le traitement n'a pas été modifié. En 1998, Wahl [17] a rapporté dans une série de 2014 interventions de chirurgie buccale effectuées sur 774 patients différents, $12(1,5 \%)$ cas d'hémorragie sévère essentiellement dus à une hypocoagulation excessive. Dans une récente étude française, Gaudy [27] a montré que sur 2384 patients traités par AVK et chez lesquels des extractions dentaires ont été réalisées en maintenant le traitement $A V K$ et en réalisant une hémostase locale préconisée par la SFMBCB, il n'y pas eu d' accident hémorragique grave. Dans 34 cas $(1,4 \%)$, une ré-intervention locale a quand même été nécessaire : il s'agissait le plus souvent d'une compression avec une compresse imbibée d'acide tranexamique ou d'adjonction de colle biologique, en ambulatoire.

Les dernières recommandations relatives à la prise en charge des patients sous AVK ont été rédigées par la SFMBCB en 2006; ces recommandations n'ont donc pas été enseignées aux praticiens sortis du cycle universitaire avant cette date. L'information est néanmoins parvenue à un certain nombre de praticiens puisque $16,5 \%$ d'entre eux prenant en charge ces patients maintiennent le traitement hypocoagulant. Mais seuls $11,2 \%$ des praticiens respectent le protocole de la SFMBCB dans sa totalité, c'est-à-dire : maintien du traitement AVK et hémostase de niveau 1 avec comblement intraalvéolaire, sutures et compression intermittente. Il existe encore un grand nombre de praticiens qui réalisent l'arrêt du traitement AVK ou un relais héparinique car l'attitude à adopter n'est pas connue de tous. Cette étude permet toutefois de remarquer que les praticiens réalisant l'arrêt du traitement s'estiment les moins bien informés sur les recommandations de la SFMBCB et sont les plus demandeurs pour une formation relative à cette prise en charge. A l'inverse, les praticiens ne stoppant pas le traitement AVK s'estiment les mieux renseignés : ils sont donc les moins demandeurs de formation. Ceci confirme que l'information n'est pas encore parvenue à l'ensemble des chirurgiens dentistes.

Tous les intervenants (chirurgien dentiste, médecin généraliste ou spécialiste, anesthésiste) devront adopter la même pratique car la responsabilité du choix thérapeutique appartient à l'ensemble de ces acteurs. Pour illustrer cette responsabilité, on peut rapporter le cas suivant. En 2007, un patient de 78 ans traité par AVK pour arythmie cardiaque et fibrillation auriculaire doit être opéré pour une cataracte invalidante. Son traitement hypocoagulant est arrêté. La mauvaise coordination entre les praticiens a fait que le traitement AVK a été repris seulement 48 heures après l'intervention. Au $3^{\text {ème jour }}$ postopératoire, le patient a présenté un accident vasculaire cérébral sévère avec perte complète de l'autonomie; son INR était à 1,7 lors de l'admission. Un an plus tard, le patient est décédé. Les enfants du patient ont engagé une procédure civile. A la vue du manque de coordination entre les différents spécialistes, les experts ont retenu une responsabilité intégrale et collective [28].

Une bonne organisation et une bonne communication entre les différents spécialistes soignants sont donc nécessaires pour mettre en pratique les dernières recommandations. L'information relative à la prise en charge des patients sous traitement AVK doit donc continuer à être largement diffusée pour pouvoir atteindre l'ensemble des praticiens. En Ille-etVilaine, 30,3\% des praticiens s'estiment être suffisamment informés sur les dernières recommandations et $63,7 \%$ pensent avoir besoin d'une formation complémentaire, preuve que l'information n'est pas parvenue à tous les praticiens. 43,3\% des praticiens ne prennent pas en charge ces patients soit qu'ils n'ont pas le plateau technique pour réaliser des actes de chirurgie buccale, soit qi'ils redoutent le risque hémorragique ou le risque thromboembolique. Parmi ceux-ci, beaucoup d'entre eux ne connaissent pas les dernières recommandations $(80,8 \%)$, mais paradoxalement peu d'entre eux souhaitent une formation continue sur ce sujet $(5,4 \%)$.

Cette étude a par ailleurs révélé une différence significative dans la prise en charge de ces patients hypocoagulés selon qu'il s'agissait de praticiens de sexe féminin ou masculin. 
Les praticiens femmes sont plus nombreux que les praticiens hommes à prendre en charge les patients sous traitement AVK ; cette différence est significative : 73,5\% contre 38,6\%. Elles réalisent les différents traitements (arrêt, relais et pas de modification du traitement AVK) dans les mêmes proportions que les hommes et le nombre de complications rencontrées par les praticiens femmes est également retrouvé dans les mêmes proportions que chez les praticiens hommes. Cependant, il existe une différence significative sur la connaissance et le besoin d'une formation. Les praticiens femmes s'estiment mieux informées sur les recommandations de la SFMBCB et estiment avoir moins besoin de formation relative à la prise en charge des patients sous traitement AVK que les praticiens hommes. Nous pouvons supposer que les femmes prendraient plus en charge les patients hypocoagulés que les hommes car elles s'informeraient plus qu'eux et connaîtraient ainsi mieux les risques hémorragiques et thromboemboliques encourus.

\section{Conclusion}

Jadis il n'y avait pas de protocole clairement établi pour la prise en charge des patients sous traitement AVK avant un acte de chirurgie buccale. En 2006, la SFMBCB et la SFC ont décidé de proposer, à partir d'une analyse critique de la littérature, des recommandations pour la prise en charge de ces patients. Il est maintenant établi que l'on ne doit pas modifier le traitement AVK avant des extractions dentaires si l'INR est stable et inférieur à 3 , pour une prise en charge en secteur de ville. Cette attitude thérapeutique doit être adoptée par l'ensemble de l'équipe pluridisciplinaire : médecin traitant, spécialiste et odontologiste. Un INR doit être réalisé dans les 48 heures précédant l'intervention et le chirurgien dentiste a l'obligation de réaliser une hémostase locale telle qu'elle est décrite dans les recommandations de la SFMBCB : matériaux de comblement intra-alvéolaire, suture et compression intermittente à l'Exacy $l^{\circledR}$.

Il est maintenant clairement démontré que le relais et l'arrêt du traitement AVK comporte un risque thromboembolique et entraînent un surcoût; le rapport coût/sécurité est positif si les recommandations de la SFMBCB sont mises en œuvre.

Cette étude dresse un premier état des lieux en Ille-etVilaine. Les recommandations de la SFMBCB et de la SFC ont déjà été adoptées par de nombreux praticiens. Cependant, les anciennes pratiques perdurent encore et il semble important que les différents acteurs de la profession prennent une part active à la diffusion et l'enseignement de ces recommandations.

\section{Références}

1. Campbell JH, Alvarado F, Murray RA. Anticoagulation and minor oral surgery: should the anticoagulation regimen be altered? J Oral Maxillofac Surg 2000;58:131-5; discussion 5-6.

2. Della Valle A, Sammartino G, Marenzi G, Tia M, Espedito di Lauro A, Ferrari F, Lo Muzio L. Prevention of postoperative bleeding in anticoagulated patients undergoing oral surgery: use of plateletrich plasma gel. J Oral Maxillofac Surg 2003;61:1275-8.

3. Persac S, Boland FX, Lavis JF, Tardif A. Tooth extraction and anticoagulants. Rev Stomatol Chir Maxillofac 2007;108:189-92.

4. Société Francophone de Médecine Buccale et de Chirurgie Buccale. Recommandations pour la prise en charge des patients sous traitement anti-vitamines $\mathrm{K}$ en chirurgie bucco-dentaire. Med Buccale Chir Buccale 2006;12:187-212.

5. Devani P, Lavery KM, Howell CJ. Dental extractions in patients on warfarin: is alteration of anticoagulant regime necessary ? $\mathrm{Br}$ J Oral Maxillofac Surg 1998;36:107-11.

6. Dunn AS, Turpie AG. Perioperative management of patients receiving oral anticoagulants: a systematic review. Arch Intern Med 2003;163:901-8.

7. Bloomer CR. Excessive hemorrhage after dental extractions using low-molecular-weight heparin (Lovenox) anticoagulation therapy. J Oral Maxillofac Surg 2004;62:101-3.

8. Dinca $S$, Arreto CD, Wierzba CB. Médicaments des troubles cardiovasculaires. Actual Odontostomatol 2003;221:39-49.

9. Chow KM, Szeto CC. Oral anticoagulant and dental procedures. Arch Intern Med 2003;163:2532; author reply 3.

10. Levesque $H$, Borg JY. Les centres de suivi et de conseil des traitements anticoagulants : un outil pour déterminer le nombre de complications hémorragiques des antivitamines K. Rev Méd Int 2003;24:75-7.

11. Oehlert WH. Dental extractions and gum surgery on anticoagulated patients. J Okla Dent Assoc 2004;94:6.

12. Pernod G, Barro C, Blanc-Jouvan F, Polack B. Routine preoperative evaluation of hemostasis. Rev Stomatol Chir Maxillofac 2003;104:91-7.

13. Perry DJ, Noakes TJ, Helliwell PS. Guidelines for the management of patients on oral anticoagulants requiring dental surgery. $\mathrm{Br}$ Dent J 2007;203:389-93.

14. Prescrire (Rédaction). Antivitamines $\mathrm{K}$ et extractions dentaires : peu de risques avec un INR inférieur à 4. Rev Prescrire 2004;22:614-8.

15. Salam S, Yusuf $H$, Milosevic A. Bleeding after dental extractions in patients taking warfarin. Br J Oral Maxillofac Surg 2007; 45:463-6.

16. Todd DW. Anticoagulated patients and oral surgery. Arch Intern Med 2003;163:1242.

17. Wahl MJ. Dental surgery in anticoagulated patients. Arch Intern Med 1998;158:1610-6.

18. Zanon E, Martinelli F, Bacci C, Cordioli G , Girolami A. Safety of dental extraction among consecutive patients on oral anticoagulant treatment managed using a specific dental management protocol. Blood Coagul Fibrinolysis 2003;14:27-30.

19. Zusman SP, Lustig JP, Bin Nun G. Cost evaluation of two methods of post tooth extraction hemostasis in patients on anticoagulant therapy. Community Dent Health 1993;10:167-73.

20. Carter G, Goss AN, Lloyd J, Tocchetti R. Current concepts of the management of dental extractions for patients taking warfarin. Aust Dent J. 2003;48:89-96; quiz 138. 
21. Borea G , Montebugnoli L, Capuzzi P, Magelli C. Tranexamic acid as a mouthwash in anticoagulant-treated patients undergoing oral surgery. An alternative method to discontinuing anticoagulant therapy. Oral Surg Oral Med Oral Pathol 1993;75:29-31.

22. Ferrieri GB, Castiglioni S, Carmagnola D, Cargnel M, Strohmenger $\mathrm{L}$, Abati S. Oral surgery in patients on anticoagulant treatment without therapy interruption. J Oral Maxillofac Surg 2007;65:1149-54.

23. Blinder D, Manor Y, Martinowitz U, Taicher S. Dental extractions in patients maintained on oral anticoagulant therapy: comparison of INR value with occurrence of postoperative bleeding. Int J Oral Maxillofac Surg 2001;30:518-21.

24. Blinder D, Manor Y, Martinowitz U, Taicher S, Hashomer T. Dental extractions in patients maintained on continued oral anticoagulant: comparison of local hemostatic modalities. Oral Surg Oral Med Oral Pathol Oral Radiol Endod 1999;88:137-40.
25. Conan M. Etude du rapport coût /sécurité lors de la prise en charge des patients sous antivitamines $\mathrm{K}$ en chirurgie buccale. Thèse de Doctorat en Chirurgie dentaire. Université de Rennes 1, 2007.

26. Laupacis A, Albers G, Dalen J, Dunn MI, Jacobson AK, Singer DE. Antithrombotic therapy in atrial fibrillation. Chest 1998;114:579S-89S.

27. Gaudy JF, Arreto CD. Anticoagulants et extractions dentaires. Arch Mal Coeur Vaiss 2005;98:859-66.

28. Gaultier C. Interruption des AVK en periopératoire. Manque de concertation entre les praticiens. Concours Méd 2007;129: 344-5. 